\title{
Three-dimensional electrostatic interactions in dynamic force microscopy: Experiment and theory
}

\author{
T. König, L. Heinke, G. H. Simon, and M. Heyde* \\ Fritz-Haber-Institut der Max-Planck-Gesellschaft, Faradayweg 4-6, D-14195 Berlin, Germany \\ (Received 24 September 2010; revised manuscript received 21 December 2010; published 25 May 2011)
}

\begin{abstract}
The measurand in dynamic force microscopy is the frequency shift, which is a direct function of the tip-sample interaction. In this work, this interaction is experimentally investigated in the parameter space of tip-sample distance and bias voltage. This three-dimensional database is theoretically described by a simple model of a Lennard-Jones potential superimposed with an electrostatic potential. The detailed study of the tip-sample interaction is essential for many scanning probe measurements and offers insight, e.g., into charge states of defects which are supposed to dominate the surface chemistry of many materials.
\end{abstract}

DOI: 10.1103/PhysRevB.83.195435

PACS number(s): 68.37.Ps, 68.37.Ef, 07.79.Cz, 07.79.Lh

\section{INTRODUCTION}

The electronic properties of surfaces down to point defect resolution have an important impact on all kinds of surface phenomena. On conducting materials, scanning tunneling microscopy (STM) can probe the electronic structure, i.e., the local density of states (LDOS), with atomic resolution. However, on insulating surfaces the situation becomes more difficult. A technique enabling measurements on insulators with very high local resolution is dynamic force microscopy (DFM). This technique is also known as noncontact atomic force microscopy (NC-AFM) or frequency modulation atomic force microscopy (FM-AFM). Although DFM cannot map the density of electron states directly, the electronic signatures and/or electronic effects on the surface can be correlated with the force between tip and sample. ${ }^{1-5}$ In careful experimental works, the frequency shift and therewith the force is studied either as a function of the tip-sample distance (Refs. 6-10) or as a function of the tip-sample bias voltage (Refs. 8,11,12). Several electronic properties have been determined with very high resolution; e.g., band-structure-related phenomena have been observed on semiconductors, ${ }^{13}$ and electron charging of quantum dots, ${ }^{11}$ adsorbates, ${ }^{14}$ and point defects ${ }^{15}$ have been detected.

In the present paper, force-distance and force-bias voltage spectroscopies are combined, showing the frequency shift as a three-dimensional function of the bias voltage and the tip-sample distance. Similar to the introduction of the threedimensional atomic force microscopy, ${ }^{16,17}$ new insights from this more-dimensional spectroscopy are expected.

\section{EXPERIMENTAL SETUP}

The measurements have been performed with a custombuilt dual-mode dynamic force microscope (DFM) and scanning tunneling microscope (STM) operating in ultrahigh vacuum at $5 \mathrm{~K}$ (Fig. 1). In the DFM mode, images are recorded by regulating the tip height to obtain a constant frequency shift of the tuning fork, to which the tip is attached. In the STM mode, the tip height is regulated to gain a fixed tunneling current. Tunneling current and frequency shift can be recorded simultaneously and the feedback can be switched between both modes instantly. This means DFM and STM measurements of exactly the same position on the surface can be performed with the same microscopic tip configuration. This constitutes the great advantage of this dual-mode setup. The tip in use for both channels is a solid Pt/Ir tip. More details about the setup can be found in Refs. 18-20. In the following we confine ourselves to the DFM signal since only DFM is sensitive to the forces acting between tip and sample.

The sample system used is epitaxially grown thin-film $\mathrm{MgO}$ on $\mathrm{Ag}(001)$. More details about the samples and their preparation can be found in Refs. 15,21. Full-metal tips and metal-supported thin films allow optimal potential control.

\section{THEORY}

The potentials and forces acting on the tip in DFM causing the shift of the resonance frequency are modeled by a LennardJones potential which is superimposed by the electrostatic interaction. ${ }^{11}$ For a thin (oxide) film on a conducting substrate, the electrostatic interaction may be modeled by considering the capacities between film and substrate and between film and tip. ${ }^{11,22}$ The potential energy $E_{\text {total }}$ is given by

$$
\begin{aligned}
E_{\mathrm{total}}= & E_{\mathrm{LJ}}+E_{e l} \\
= & 4 \epsilon\left(\left(\frac{\sigma}{z}\right)^{12}-\left(\frac{\sigma}{z}\right)^{6}\right) \\
& +\frac{q^{2}}{2 C_{\Sigma}(z)}-\frac{C_{\mathrm{tip}}(z)}{C_{\Sigma}(z)} q V-\frac{1}{2} \frac{C_{\mathrm{sub}} C_{\mathrm{tip}}(z)}{C_{\Sigma}(z)} V^{2} .
\end{aligned}
$$

The Lennard-Jones potential is determined by the parameters $\epsilon$ and $\sigma$, corresponding to the depth of the potential well and the distance at which the repulsive and attractive parts balance each other, respectively. The equilibrium distance of the Lennard-Jones potential is $\sqrt[6]{2} \sigma$. The attractive $z^{-6}$ term is also called the van der Waals term.

The electrostatic interaction is determined by the charge $q$ in the surface, the voltage between tip and sample $V_{\text {bias }}$, and the capacities, where $C_{\text {tip }}(z), C_{\text {sub }}$, and $C_{\Sigma}(z)$ denote the capacities between the tip and the thin film and between the thin film and the substrate, as well as the sum of both, respectively. $z$ is the distance between the tip and the sample surface. Furthermore, $V=V_{\text {bias }}-\Delta \Phi / e$ with $V_{\text {bias }}$ denoting the applied bias voltage and $\Delta \Phi$ the contact potential, i.e., the work function difference between tip and surface, and $e$ the elementary charge. 
(a)

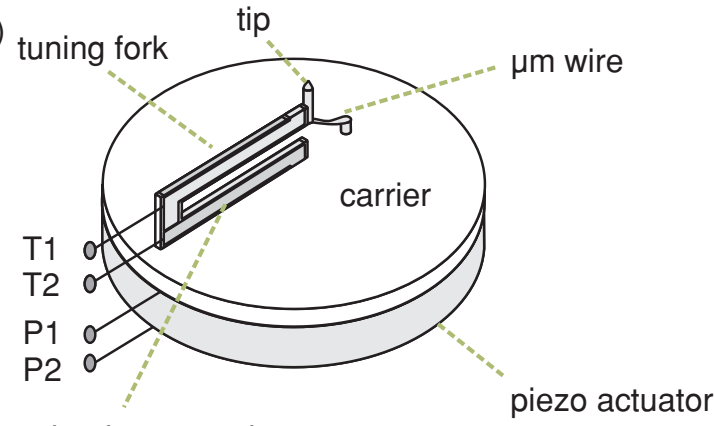

prong glued onto carrier
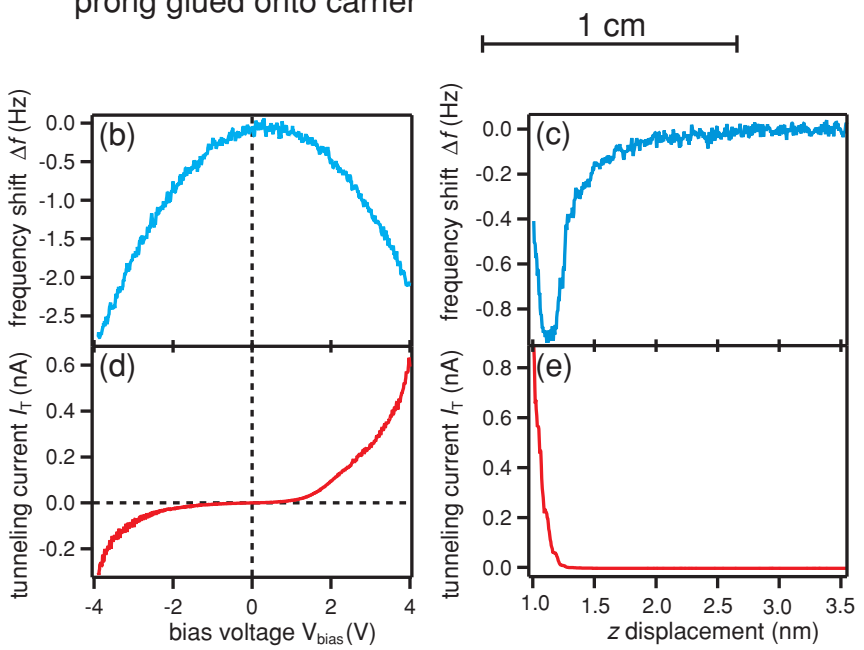

FIG. 1. (Color online) Dual-mode setup. (a) Sketch of the dualmode sensor. The DFM/STM tuning-fork sensor, to which the tip is connected, is glued onto the MACOR carrier. DFM is performed by detecting the signal from the tuning fork via contacts T1 and T2. P1 and $\mathrm{P} 2$ are the contacts of the excitation piezo, which excites vibration of the tuning fork. By means of the $\mu \mathrm{m}$ wire, the tunneling current can be recorded. With this setup, the frequency shift and the tunneling current can be recorded simultaneously with the same microscopic tip configuration. This enables the spectroscopy of the frequency shift vs bias voltage (b) and vs $z$ displacement (c), as well as the tunneling current vs bias voltage (d) and vs $z$ displacement (e).

The force $F$ between tip and sample follows by the gradient of the potential energy; i.e.,

$$
\begin{aligned}
F_{\text {total }}= & 4 \epsilon\left(-\frac{12}{z}\left(\frac{\sigma}{z}\right)^{12}+\frac{6}{z}\left(\frac{\sigma}{z}\right)^{6}\right) \\
& -\frac{q^{2}}{2} \frac{\frac{\partial C_{\Sigma}}{\partial z}}{C_{\Sigma}^{2}}-\frac{\frac{\partial C_{\text {tip }}}{\partial z} C_{\Sigma}-C_{\text {tip }} \frac{\partial C_{\Sigma}}{\partial z}}{C_{\Sigma}^{2}} q V \\
& -\frac{1}{2} C_{\text {sub }} \frac{\frac{\partial C_{\text {tip }}}{\partial z} C_{\Sigma}-C_{\text {tip }} \frac{\partial C_{\Sigma}}{\partial z}}{C_{\Sigma}^{2}} V^{2} \\
= & \epsilon\left(-\frac{48}{\sigma}\left(\frac{\sigma}{z}\right)^{13}+\frac{24}{\sigma}\left(\frac{\sigma}{z}\right)^{7}\right) \\
& -\frac{\partial C_{\text {series }}}{\partial z}\left(V_{\text {bias }}-\frac{\Delta \Phi}{e}-\frac{q}{C_{\text {sub }}}\right)^{2},
\end{aligned}
$$

where $C_{\text {series }}=C_{\text {tip }} C_{\text {sub }} /\left(C_{\text {tip }}+C_{\text {sub }}\right)$, which is the capacity of the series connection of the substrate-film and film-tip capacitors. If we consider the system of a (nonconducting) thin oxide film, which may contain localized charges, on a (conducting) metal support and a (conducting) metal tip, the contact potential between tip and sample and the charge in the surface can be combined to an effective contact potential $\Delta \Phi_{\text {eff }}$; i.e., $\Delta \Phi_{\text {eff }}=\Delta \Phi-n e^{2} / C_{2} \cdot{ }^{23}$ This results in

$$
\begin{aligned}
F_{\text {total }}= & \epsilon\left(-\frac{48}{\sigma}\left(\frac{\sigma}{z}\right)^{13}+\frac{24}{\sigma}\left(\frac{\sigma}{z}\right)^{7}\right) \\
& -\frac{\partial C_{\text {series }}}{\partial z}\left(V_{\text {bias }}-\frac{\Delta \Phi_{\text {eff }}}{e}\right)^{2} .
\end{aligned}
$$

The frequency shift $\Delta f$ of the tuning fork follows from the force field with ${ }^{24}$

$$
\Delta f=-\frac{f_{0}}{\pi a k} \int_{-1}^{1} F(z+a(1+u)) \frac{u}{\sqrt{1-u^{2}}} d u ;
$$

$f_{0}$ denotes the unperturbed resonance frequency, $a$ the amplitude, and $k$ the spring constant of the tuning fork. Note, $z$ denotes the minimum distance between the surface and the oscillating tip. In Fig. 2, the frequency shift is calculated as a function of the tip-sample distance $z$ and the bias voltage $V_{\text {bias }}$. The superposition of the Lennard-Jones potential and of the parabolic dependence on the bias voltage are clearly visible. The size of the amplitude slightly changes the $\Delta f$ curve.$^{24} \Delta f$ converges to the gradient of the force for an infinitesimally small amplitude.

\section{RESULTS AND DISCUSSION}

Apart from determining the topography of the surface termination with atomic resolution, DFM can also be used for spectroscopy as shown in Figs. 1(b) and 1(c). Due to the relation between the recorded frequency shift and the force [see Eq. (4)], this spectroscopy is often called force spectroscopy. At a constant $(x, y)$ position, the experimental data can be recorded either at a constant tip-sample distance measuring the frequency shift with respect to bias voltage or at a constant bias voltage detecting the frequency shift as a function of tip-sample distance. Both spectroscopies are important for a detailed analysis and understanding of the tip-sample forces. Therefore, a combination of both spectroscopies as theoretically presented in Fig. 2 is especially desirable. During all the measurements a very stable tip configuration has to be ensured since the measurements have to be performed with the very same microscopic tip.

Experimental data showing the three-dimensional tipsample interaction in the parameter space of $z$ displacement and bias voltage $V_{\text {bias }}$ are presented in Fig. 3. To the authors' knowledge, this is the first experimental presentation of the frequency shift in this parameter space. Note that only the $z$ displacement is experimentally accessible and the exact distance between tip and sample cannot be determined by DFM directly. However, the tip-sample distance equals the $z$ displacement plus an offset.

Cuts of Fig. 3 along $z$ at different constant bias voltages $V_{\text {bias }}$ are shown in Fig. 4(a). With increasing absolute voltages the graphs show a stronger tip-sample interaction since $F_{\mathrm{el}}$ increases according to Eq. (3). Figure 4(b) shows cuts of Fig. 3 along $V_{\text {bias }}$ at different constant $z$ positions. The quadratic dependence with the maximum at the effective 
(a)
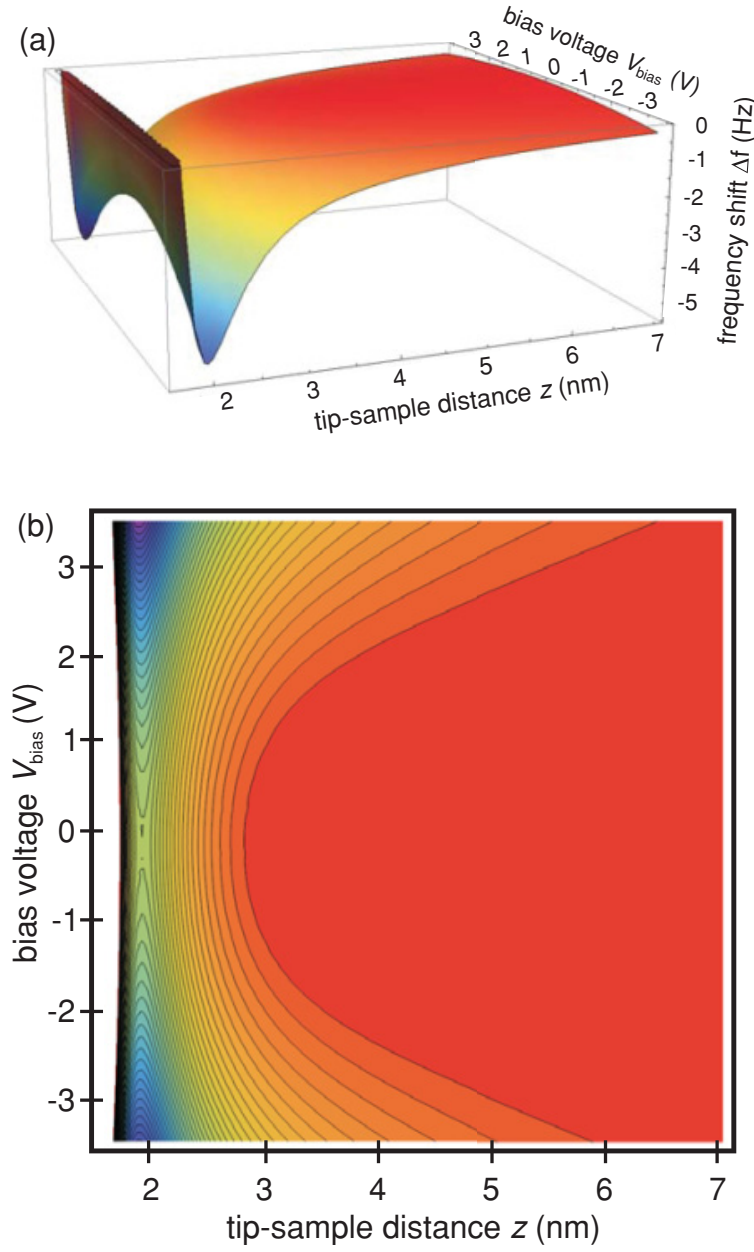

FIG. 2. (Color online) Frequency shift as a function of the bias voltage $V_{\text {bias }}$ and of the tip-sample distance $z$ as following from Eqs. (3) and (4). (a) Surface plot and (b) contour plot. The Lennard-Jones parameters are $\epsilon=2.8 \times 10^{-18} \mathrm{~J}$ and $\sigma=1.6 \mathrm{~nm}$ and the effective contact potential is $\Delta \Phi_{\text {eff }}=-0.15 \mathrm{eV}$. A sphere with a radius of $95 \mathrm{~nm}$ in front of a plane wall (Ref. 25) is used to model the capacity $C_{\text {series }}$. The amplitude of the tuning fork is $a=3 \times 10^{-10} \mathrm{~m}$, the spring constant is $k=21 \mathrm{kN} \mathrm{m}^{-1}$, and the unperturbed resonance frequency is $f_{0}=21 \mathrm{kHz}$.

contact potential is clearly visible. The method of determining the contact potential from this parabola is widely known as Kelvin probe force microscopy. ${ }^{27}$

The experimental data and the theory [Eqs. (4) and (3) with the parameters given in Fig. 2] are in very good agreement, as shown in Fig. 4. During the data analysis an additional van der Waals term to compensate for the influence of the tip configuration ${ }^{28}$ superimposed by the electrostatic interaction has been tried out not leading to a significantly better agreement. This means the simple theoretical model of a Lennard-Jones potential superimposed by the electrostatic interaction is sufficient for describing the experimentally determined tip-sample interaction as a function of the bias voltage and the tip-sample distance.

Note that there is very good agreement between theory and experiment for the whole data set shown in Fig. 3, not only for the plots in Fig. 4. However, due to the large amount of adjustable parameters, it may be that the determined
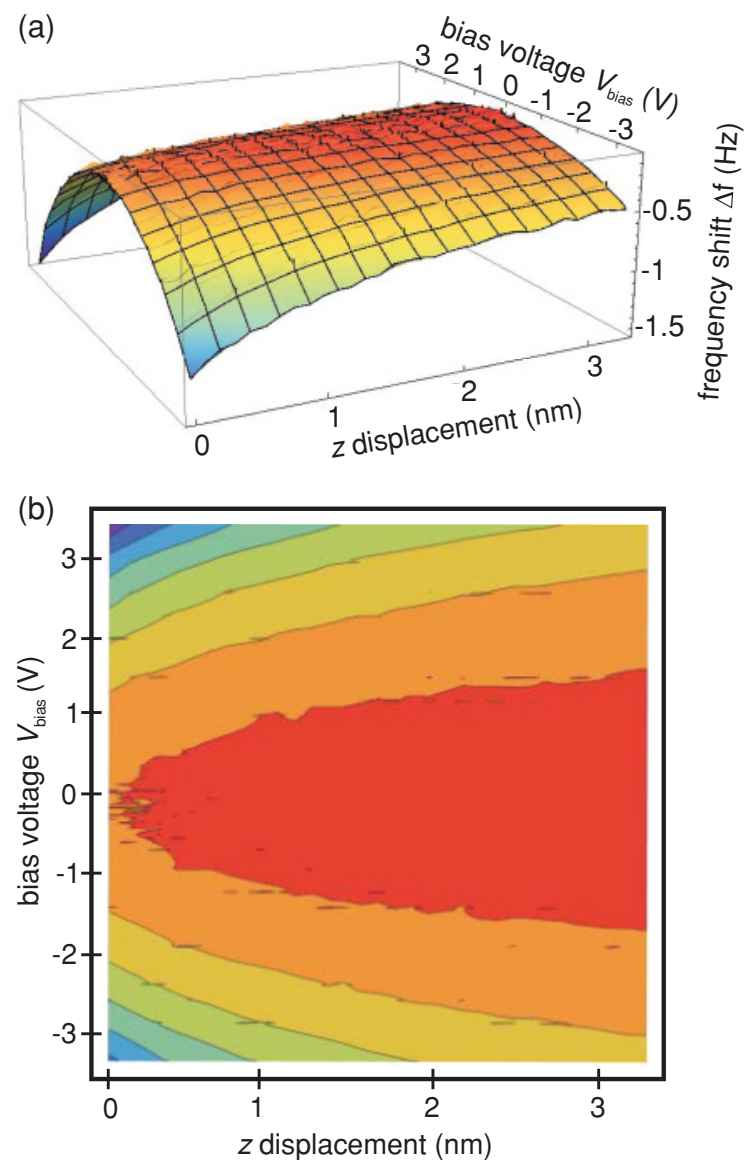

FIG. 3. (Color online) Frequency shift experimentally determined by DFM as a function of the bias voltage $V_{\text {bias }}$ and the $z$ displacement. (a) Surface plot and (b) contour plot. The amplitude of the tuning fork is $a=3 \times 10^{-10} \mathrm{~m}$, the spring constant is $k \approx$ $21 \mathrm{kN} \mathrm{m}^{-1}$, and the unperturbed resonance frequency is $f_{0}=21 \mathrm{kHz}$ (Ref. 26).

parameters shown in Fig. 2 represent only a local optimum. This means a parameter set which represents the experimental data even better cannot be excluded.

The interaction in Fig. 4(b) shows a parabolic, always attractive behavior, as predicted by the quadratic dependence in Eq. (3). If the $z$ position is constant and the voltage swept, only the electrostatic force varies. The contribution from the Lennard-Jones interaction, which is constant, leads to a vertical offset. The desired information about local electronic properties is now included in the shift of the parabola along the abscissa. The shift results from the local effective contact potential $\Delta \Phi_{\text {eff }}$ [see Eq. (3)]. The local effective contact potential is significantly affected by local charges as for example present in charged adsorbates ${ }^{29}$ or defect sites. ${ }^{15,30}$ The local variation in the effective contact potential can then be used to identify the charge state of single point defects on a local scale. ${ }^{15}$ This fact is the basis for the results cited in the introduction.

Changes of the effective contact potential are determined by measuring changes of the maximum of the frequency shift with respect to bias voltage at a constant $z$ position. Such measurements are shown in Fig. 5. The green (light gray) parabola represents typical data taken on the bare $\operatorname{Ag}(001)$ 

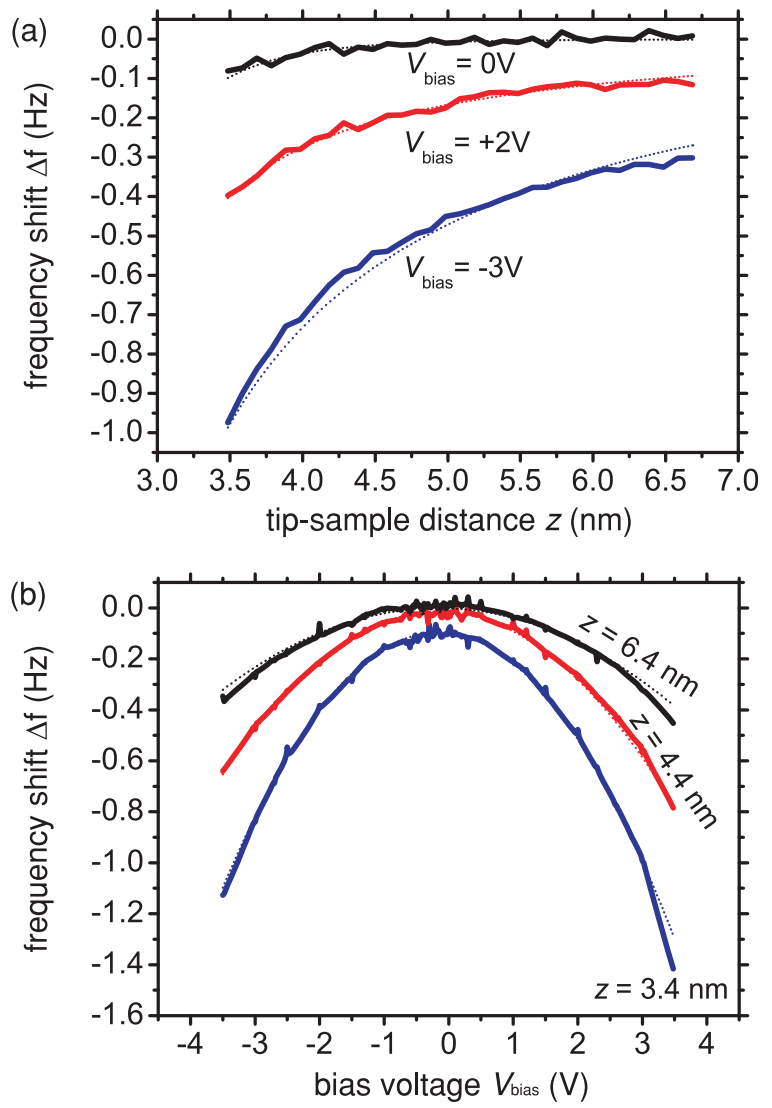

FIG. 4. (Color online) Frequency shift vs (a) the tip-sample distance and (b) the bias voltage. The experimental data (thick lines) correspond to cuts through Fig. 3. The thin dotted lines are calculated by Eqs. (4) and (3) with the parameters shown in the caption of Fig. 2. An experimentally recorded $z$ displacement of 0 corresponds to a tip-sample distance of $z=3.4 \mathrm{~nm}$.

surface. The shift with respect to the zero bias voltage is given by $\Delta \Phi_{1}=\Phi_{\mathrm{Ag}}-\Phi_{\text {tip }}$, where $\Phi_{\mathrm{Ag}}$ and $\Phi_{\text {tip }}$ are the work functions of the bare $\operatorname{Ag}(001)$ surface and the tip, respectively. The red parabola in Fig. 5 has been measured on a $3 \mathrm{ML} \mathrm{MgO}$ film grown on $\operatorname{Ag}(001) .{ }^{15}$ The shift with respect to zero bias voltage is given by $\Delta \Phi_{2}=\Phi_{\mathrm{MgO} / \mathrm{Ag}}-\Phi_{\text {tip }}$, where $\Phi_{\mathrm{MgO} / \mathrm{Ag}}$ is the work function of the $\mathrm{Ag}(001)$ surface with $3 \mathrm{ML} \mathrm{MgO}$ grown on top. From these two measurements the shift of the work function can be determined ${ }^{21}$ as

$$
\Delta \Phi=\Delta \Phi_{2}-\Delta \Phi_{1}=\Phi_{\mathrm{MgO} / \mathrm{Ag}}-\Phi_{\mathrm{Ag}} .
$$

Thus the work function of the tip cancels and the work function shift induced by the $\mathrm{MgO}$ overlayer can be derived. ${ }^{21}$ This results in a reduction of the work function by $1.2 \mathrm{eV}$ due to the oxide film on $\mathrm{Ag}(001)$.

Care has to be taken when the investigated element (defect, atom) is smaller than the integration area determined by the tip radius and tip-sample distance. Since the electrostatic forces are long range in character, an integration is always present causing an averaging. For features smaller than the integration area, which can be in the range of roughly $20 \mathrm{~nm}^{2}$ (see Ref. 30) to some $1000 \mathrm{~nm}^{2}$, the height between measurements at different sites should be constant, since an increase of the

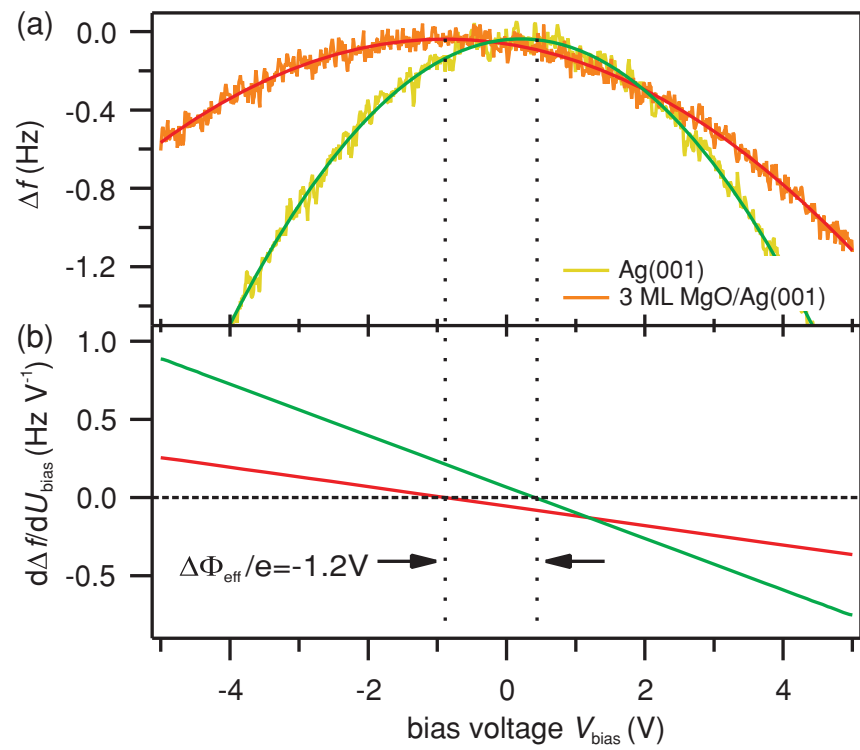

FIG. 5. (Color online) (a) Frequency shift with varying bias voltage at fixed tip-sample distance on $\mathrm{Ag}(001)$ and $3 \mathrm{ML} \mathrm{MgO/Ag(001).}$ The indicated effective contact potential shift of $\Delta \Phi_{\text {eff }}=-1.2 \mathrm{eV}$ results from the shift of the $\operatorname{Ag}(001)$ work function induced by the $\mathrm{MgO}$ overlayers, reducing the surface dipole. Graph (b) shows the derivatives of the parabolic fits to the data.

tip-sample distance results in an increase of the integration area. $^{21}$ That means, the maximum positions of the parabola for different $z$ displacements is not constant and may shift.

The effect of the tip-sample distance on the resolution is shown in Fig. 6. Based on tip-sample pair potential interactions the contribution of the tip atom closest to the surface with respect to the full tip-sample interaction has been analyzed. The contribution of the foremost tip atom to the full interaction force increases with decreasing tip-sample interaction resulting in a rise in local resolution. The calculation is based on the following equation:

$$
\begin{aligned}
F_{\mathrm{ts}}(z) & =-\frac{\partial V_{\mathrm{ts}}}{\partial z}=-\frac{\partial}{\partial z}\left(V_{j}+\sum_{m \neq j}^{N} V_{m}\right) \\
& =F_{j}(z)+\frac{q_{\mathrm{tip}}}{4 \pi \epsilon_{0}} \sum_{m \neq j}^{N} \frac{q_{\mathrm{sample}, m}}{r_{m}^{2}} \frac{z}{r_{m}},
\end{aligned}
$$

where $q_{\text {tip }}$ and $q_{\text {sample, } m}$ are the charges of the tip and the sample atom $m$ and $r_{m}$ is the distance between tip and sample atom m. $F_{j}$ is the force between tip and the atom nearest to the tip. Thus for small distances the local resolution increases; i.e., for atomic resolution or defect analysis the tip-sample distance should be as small as possible. However, care has to be taken since tip changes are very likely at strong tip-sample interaction, occurring at close distances and high voltages. Similar results are obtained for different geometries; see, e.g., Ref. 31.

The optimization of local resolution is not only necessary for spectroscopic measurements. The electrostatic force affects also the resolution during lateral scans at constant frequency shift and therewith at constant tip-sample interaction. The (black) contour lines in Figs. 2(b) and 3(b) are examples 


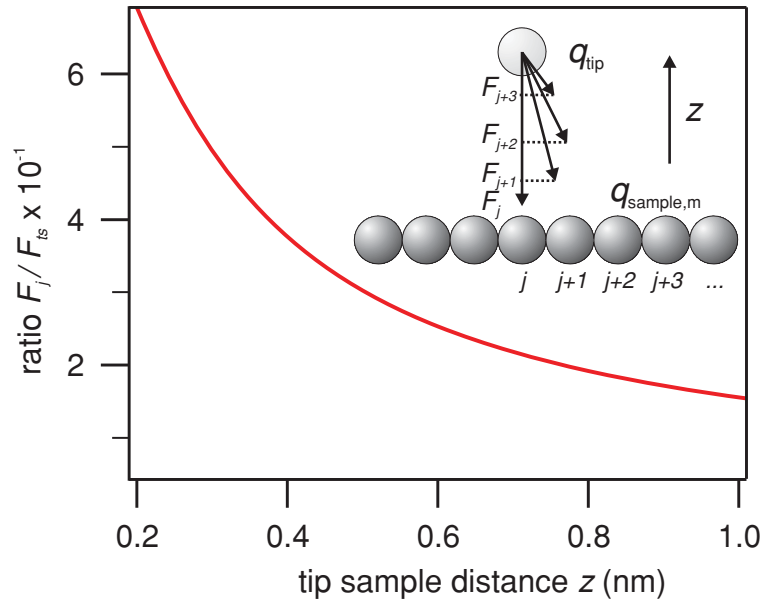

FIG. 6. (Color online) Ratio of the tip-sample interactions resulting from the atom directly beneath the tip (atom $j$ ) and the full tip-sample interaction of all surface atoms with the tip $F_{\mathrm{ts}}$. The inset shows the tip-sample arrangement.

for possible $\Delta f$ set points. Following such a contour means constant frequency shift (as well as constant tip-sample interaction). However, note that the resolution for local imaging is not constant along such a contour. For a given frequency shift, the tip-sample distance is always the smallest when $V_{\text {bias }}$ equals $\Delta \Phi_{\text {eff }} / e$; i.e., when the effective contact potential is compensated and thus electrostatic forces $F_{\text {el }}$ equal zero. This is a prerequisite for setting the best suited set point of the frequency shift and the bias voltage enabling DFM images with true atomic ${ }^{32}$ or submolecular ${ }^{33}$ resolution. This demonstrates that knowing the contact potential is essential for DFM and plots such as Figs. 2 and 3 are crucial for a better understanding of the tip-sample interaction.

\section{SUMMARY}

The frequency shift $\Delta f$ in the parameter space of the tip-sample distance $z$ and the bias voltage $V_{\text {bias }}$ are measured. These three-dimensional experimental data can be well described by a Lennard-Jones potential superimposed with an electrostatic interaction. Cuts along constant bias voltages show the force-distance curves. Information about electronic properties of the surface can be derived at constant tip-sample distances, showing a quadratic dependence of the force on the bias voltage. By comparing parabolas of different surface sites, electrostatic information down to point defect resolution can be gained. The resolution depends on the tip-sample distance which should be constant when features smaller than the area integrated by the long-range electrostatic interaction are analyzed. A stable setup and tip are prerequisites for high-resolution measurements with point defect resolution. The electrostatic force also affects the local resolution in imaging processes and the highest resolution can be obtained by DFM when the bias voltage compensates the effective contact potential. So, this study shows that the knowledge of the tip-sample interaction is very important for high-resolution DFM.

\section{ACKNOWLEDGMENTS}

Special thanks go to H.-J. Freund for his constant support of our work. We also acknowledge technical support from $\mathrm{G}$. Thielsch and H.-P. Rust. *heyde@fhi-berlin.mpg.de

${ }^{1}$ C. J. Chen, J. Phys. Condens. Matter 3, 1227 (1991).

${ }^{2}$ A. Schirmeisen, G. Cross, A. Stalder, P. Grütter, and U. Dürig, New J. Phys. 2, 29 (2000).

${ }^{3}$ C. Loppacher, M. Bammerlin, M. Guggisberg, S. Schär, R. Bennewitz, R. B. A. Baratoff, E. Meyer, and H.-J. Güntherodt, Phys. Rev. B 62, 16944 (2000).

${ }^{4}$ T. König, G. H. Simon, H.-P. Rust, and M. Heyde, Appl. Phys. Lett. 95, 083116 (2009).

${ }^{5}$ S. Ciraci, E. Tekman, A. Baratoff, and I. P. Batra, Phys. Rev. B 46, 10411 (1992).

${ }^{6}$ M. Heyde, M. Sterrer, H. Rust, and H. Freund, Nanotechnology 17, S101 (2006) (Proceedings of the 8th International Conference on Non-Contact Atomic Force Microscopy, Bad Essen, Germany, 15-18 August 2005).

${ }^{7}$ O. Pfeiffer, L. Nony, R. Bennewitz, A. Baratoff, and E. Meyer, Nanotechnology 15, S101 (2004) (Proceedings of the 6th International Conference on Non-Contact Atomic Force Microscopy, Dingle, Ireland, 31 August-3 September, 2003).

${ }^{8}$ L. Nony, F. Bocquet, C. Loppacher, and T. Glatzel, Nanotechnology 20, 264014 (2009).

${ }^{9}$ M. Lantz, H. Hug, R. Hoffmann, P. van Schendel, P. Kappenberger, S. Martin, A. Baratoff, and H. Guntherodt, Science 291, 2580 (2001).
${ }^{10}$ Y. Sugimoto, P. Pou, M. Abe, P. Jelinek, R. Perez, S. Morita, and O. Custance, Nature (London) 446, 64 (2007).

${ }^{11}$ R. Stomp, Y. Miyahara, S. Schaer, Q. Sun, H. Guo, P. Grütter, S. Studenikin, P. Poole, and A. Sachrajda, Phys. Rev. Lett. 94, 056802 (2005).

${ }^{12}$ M. Guggisberg, M. Bammerlin, C. Loppacher, O. Pfeiffer, A. Abdurixit, V. Barwich, R. Bennewitz, A. Baratoff, E. Meyer, and H. J. Guntherodt, Phys. Rev. B 61, 11151 (2000).

${ }^{13}$ T. Arai and M. Tomitori, Phys. Rev. Lett. 93, 256101 (2004).

${ }^{14}$ L. Gross, F. Mohn, P. Liljeroth, J. Repp, F. Giessibl, and G. Meyer, Science 324, 1428 (2009).

${ }^{15}$ T. König, G. H. Simon, H.-P. Rust, G. Pacchioni, M. Heyde, and H. J. Freund, J. Am. Chem. Soc. 131, 17544 (2009).

${ }^{16}$ M. Z. Baykara, T. C. Schwendemann, E. I. Altman, and U. D. Schwarz, Adv. Mater. 22, 2838 (2010).

${ }^{17}$ B. J. Albers, T. C. Schwendemann, M. Z. Baykara, NicolasPilet, M. Liebmann, E. I. Altman, and U. D. Schwarz, Nature Nanotechnology 4, 307 (2009).

${ }^{18}$ M. Heyde, M. Kulawik, H.-P. Rust, and H.-J. Freund, Rev. Sci. Instrum. 75, 2446 (2004).

${ }^{19}$ M. Heyde, G. H. Simon, H.-P. Rust, and H.-J. Freund, Appl. Phys. Lett. 89, 263107 (2006).

${ }^{20}$ H.-P. Rust, M. Heyde, and H.-J. Freund, Rev. Sci. Instrum. 77, 043710 (2006) 
${ }^{21}$ T. König, G. H. Simon, H.-P. Rust, and M. Heyde, J. Phys. Chem. C 113, 11301 (2009).

${ }^{22} \mathrm{R}$. Waser, Nanoelectronics and Information Technology (Wiley$\mathrm{VCH}$, Weinheim, 2005).

${ }^{23}$ K.-M. Yang, J.-Y. Chung, M.-F. Hsieh, and D.-S. Lin, Jpn. J. Appl. Phys. 46, 4395 (2007).

${ }^{24}$ J. E. Sader and S. P. Jarvis, Appl. Phys. Lett. 84, 1801 (2004).

${ }^{25} \mathrm{~J}$. Crowley, in Proceedings of the ESA Annual Meeting on Electrostatics, 2008, p. D1.

${ }^{26}$ G. H. Simon, M. Heyde, and H.-P. Rust, Nanotechnology 18, 255503 (2007).

${ }^{27}$ M. Nonnenmacher, M. Oboyle, and H. Wickramasighe, Appl. Phys. Lett. 58, 2921 (1991).
${ }^{28}$ H. Hölscher, W. Allers, U. D. Schwarz, A. Schwarz, and R. Wiesendanger, Phys. Rev. B 62, 6967 (2000).

${ }^{29}$ M. Sterrer, T. Risse, U. M. Pozzoni, L. Giordano, M. Heyde, H.-P. Rust, G. Pacchioni, and H.-J. Freund, Phys. Rev. Lett. 98, 096107 (2007).

${ }^{30}$ L. Heinke, L. Lichtenstein, G. H. Simon, T. König, M. Heyde, and H.-J. Freund, Chem. Phys. Chem. 11, 2085 (2010).

${ }^{31}$ R. Feynman, R. B. Leighton, and M. Sands, The Feynman Lectures on Physics, Vol. 2 (Addison-Wesley, Reading, 1964).

${ }^{32}$ G. H. Simon, T. König, H.-P. Rust, M. Heyde, and H.-J. Freund, New J. Phys. 11, 093009 (2009).

${ }^{33}$ L. Gross, F. Mohn, N. Moll, P. Liljeroth, and G. Meyer, Science 325, 1110 (2009). 